\title{
Analysis of Open Space Types in Urban Centers Based on Functional Features
}

\author{
Junyan Yang, ${ }^{1, *}$, Fangyuan Zhang ${ }^{1}$, and Beixiang Shi ${ }^{1}$ \\ ${ }^{1}$ Southeast University, School of Architecture, Nanjing 210096, China
}

\begin{abstract}
The open space in urban centers is not only the element to beautify the landscape of urban public centers, but also bears multiple functional needs and reflects cultural values. Based on the classification system of the open space in urban centers, this paper sorts out the concept of the open space of urban centers, analyzes the evolution mode of its scale and shape, explores the way of cultivation and construction, and then puts forward a number of planning points for reasonable planning and to protect the open space in urban centers.
\end{abstract}

\section{Introduction}

Different countries or scholars have different definitions of urban open space due to different times and different research requirements.

For example, the US 1961 Housing Act stipulates that open space is any undeveloped or largely undeveloped land within an urban area. It not only has the value of parks and entertainment, and the value of land and other natural resources protection, but also the value of history or landscape ${ }^{1}$. The Japanese scholars Takahara argues that the open space is composed of two parts: public green space and private green space, emphasizing the green space of the city ${ }^{2}$. Author Alexander defines open space in Architectural Pattern Language as "Any place that makes people feel comfortable, has a natural basis, and can look into a wider space, can be called an open space" ${ }^{13}$. A. Heckscher believes that open space includes not only the natural environment in the city, such as green spaces and water, but also artificial grounds such as squares and roads ${ }^{4}$. However, H.Sbirvani defines open space as all garden landscapes, hard landscapes, parking lots, and recreational facilities in the city ${ }^{5}$.

Some scholars in China reckon that open space refers to the urban public external space, including natural scenery, squares, roads, public green space and rest space, etc $^{6}$. There are other scholars who consider that open space, on the one hand, refers to the space that is relatively open, less closed and less space-constrained, and on the other hand points to the space opened by the masses for the majority of the people. It refers not only to the landscapes like parks and green spaces, but also the streets, squares, alleys and courtyards are all within its scope $^{7}$.

\section{Open space system in urban centers}

\footnotetext{
* Corresponding author: yangjy_seu@163.com
}

The open space system in urban centers refers to the site system constructed by the open space outside the building entity in the central area of the city that provides public activities. It is an important place for people, people and nature to exchange information, material and energy.

In the central area of the city, due to the high density, large-capacity building space, dense human-car activities, scarce and increasingly depleted land, the open space system is particularly precious and is the main research object in urban planning and design. Provided site for the public activities, the open space in urban centers enable to improve the consumption quality; maintain the ecological environment, preserve the natural landscape with ecological landscape significance, maintain the coordination between the human and the natural environment, and reflect the sustainability of the environment; organically organize architectural space and activity in urban centers, exercising functions such as culture and rest; alleviating the tension of the transportation and distribution system and facilitating the ability of disaster prevention and evacuation ${ }^{8}$.

In terms of functional characteristics, the open space system in urban centers can be divided into three types: open space for leisure, for commerce and for transportation. Together, they form the dynamic atmosphere and provide a place to recreation activity and public communication for citizens.

The analysis based on different types of the open space system in urban centers is of great significance to its rational planning and design. This paper analyzes the three types for the above classification.

\section{Open space for leisure}

The open space for leisure (Green Space) is the real "green lung" in the high-density space of the urban centers. As an indispensable landscape structure, it can not only beautify the landscape, purify the air, improve 
the local microclimate, prevent noise pollution and disasters, but also provide facilities and places for leisure, entertainment, and communication for pedestrians in urban centers. The Green Space mainly include green space parks, green space squares, waterfront greenbelts and water surfaces, which have different characteristics from the general green space.

\subsection{Scale threshold of open space for leisure}

The scale indicator of open space is an important basis for measuring the effect of Green Space in urban centers. In the general urban areas, the dot-shaped Green Space is relatively small and economical, such as street green spaces and small squares scattered throughout the city. As for the urban centers, due to the complexity of the activities and the high-intensity flow of people, the open space should reach a certain scale threshold, and small green spaces are difficult to function.

The survey shows that although it has certain landscape functions, the small green space with an area of less than 1 hectare in urban centers is equipped few leisure facilities, and its ecological functions and activities are also weak. Almost no one stops there, which is caused by various reasons, such as environment quality, behavior psychology, commerce interference and ecology thresholds.

From the perspective of environment quality, due to the larger traffic flow, more noise interference and dust pollution in urban centers, small-scale green space lacks sufficient green barriers to create a feeling of leisure. From a psychological point of view, there are many three-dimensional activity platforms and high-rise buildings in urban centers, therefore, people in small green areas, where couldn't provide relatively relaxed and quiet state of mind, are prone to the tension of visual surveillance and unwilling to stay. As for actual use, owing to the pressure of commerce in urban centers, small green spaces are often occupied by commercial displays because of the lack of clear site definitions, which exacerbates the deterioration of environment quality. From the point of gardening, only if the green space area reaches 3 hectares or more, the role of purifying the air and improving the local microclimate can be exerted. Consequently, from the analysis of actual green space in many urban centers in China and abroad, in the case of the same green space rate index, the concentrated green space is much more effective than the scattered green space.

For example, from the spatial analysis of the centers of Hong Kong Island, there are more than 210 open spaces along the mountain in Central, the largest of which is 10 hectares. (Fig.1) These open spaces not only make the contour of Central "discontinuously connected", but also pay great attention to the alignment relationship with the mountain in the layout, so that people can see the ridge line in each open space, which enriches the level of the landscape. Therefore, it is popular among the public and tourists.

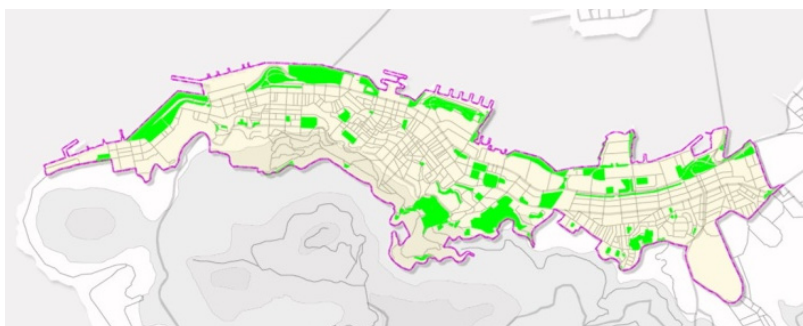

Fig. 1. Analysis of the open space distribution in Central

At present, China's planning of urban green open space mainly adopts the indicators control method of green space rate, which makes it often placed in the internal occlusion space or block corner left after the development of high-quality land. Therefore, although the total amount of statistical data meets the index of planning, the public demand for open space is difficult to meet. The large green open space model in urban centers of Singapore, Hong Kong and other megacities can be a good reference model for the planning of China.

\subsection{Compound form of open space for leisure}

The spatial form of urban centers is not homogeneous. The multiple functions of the central service, such as business, finance, and culture, are distributed in the central node space where the hard core is the agglomeration point. With high-intensity and high-density, the hard core is the area gathering a variety of function as well as a place with landmark features. There are a lot of "shadow area" around the hard core or between the multiple hard cores. In these shadow areas, commercial commerce is difficult to gather and develop, and it is close to the hard core of urban centers, which brings superior location and is convenient for the citizens to reach. It is also a great place to enjoy the iconic buildings in urban centers.

The layout of the open space for leisure should be distributed in the periphery of the hard core. Integrated with the shadow area, it is relatively concentrated into pieces, and interspersed with the building group, forming open spaces in the form of a wedge, a belt, a ring, and a sheet. From the perspective of elements, due to a large number of ventilation corridors and airflow microcirculation system formed by the large flow of people and vehicles in urban centers, the composite nesting pattern, consisting of water surface, woods, grassland and hard open square, the main and secondary roads, has stronger ecological effects, such as improving air quality and reducing noise.

In the centers of Singapore Bay-Orchard, there are nearly 100 green spaces. The area of open space outside the hard core is 2.8 square kilometers. There are large green spaces such as Farrer Park, Pearl Bank Park, Fort Canning Park, and many small patches of street green space. Most of these open spaces are located in the shadow area of the centers of the Gulf-Orchard, which not only improves the ecological environment and air quality, but also becomes the best point of appreciation for the high-rise buildings. (Fig.2) 


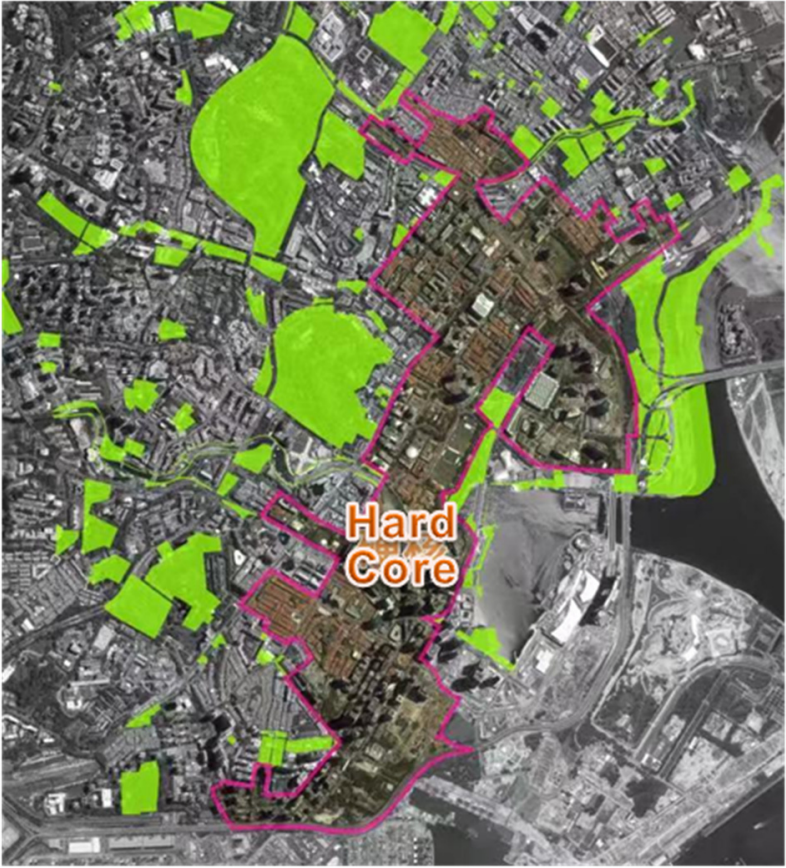

A. Open space distributed in the shadow area outside the hard core
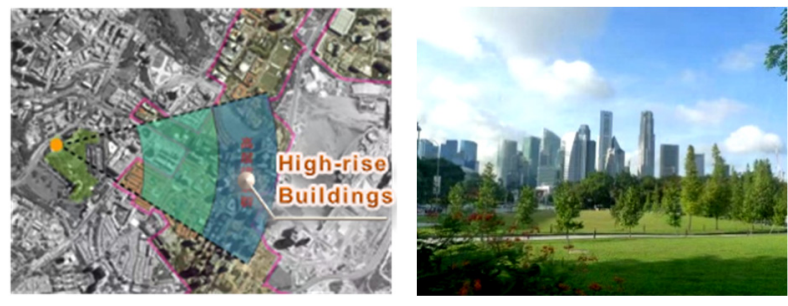

B. Central skyline from Pearl Bank Park

Fig. 2. Compound form of open space in Singapore Bay-Orchard centers

\subsection{Three-dimensional development of open space for leisure}

The three-dimensional development of Green Space in urban centers can enhance the development intensity of the land, and is essentially an urban development strategy that is in line with the intensive use of land. The squares and green spaces opened up in urban centers have a very important significance in improving the urban ecology, environment and landscape, and have a great appreciation effect on the surrounding land, but the economic value of the land itself is not full play, high demolition costs and lack of direct economic returns make the green space construction in urban centers lack economic feasibility. If the underground space can be developed at the same time, it will not only optimize the urban open space resources, but also expand the space capacity of urban centers and increase the utilization value of the high price land. The three-dimensional development of the Green Space in urban centers has the following three advantages.

First, it is conducive to expanding the capacity of public facilities in urban centers and solving the acute economic contradictions in the development of Green Space. It is an effective method to expand its underground space to set up underground commercial street, sunken square, underground parking lot, infrastructure supply, service facilities, etc.
Second, it is conducive to obtaining superior benefits of pedestrian, and solving the problem of maintenance. If the people those attracted by Green Space can guide into the underground commercial and leisure facilities, the extended consumption chain will achieve superior economic benefits. Meanwhile, underground space development reduces the supporting facilities on the ground and combines the maintenance of greening with the operators of the underground facilities, which achieves super environmental benefits.

Third, it is conducive to enriching the landscape in urban centers and improving the overall capability of disaster prevention. Filling various soils suitably for different greening plants during the underground space engineering, enables seasonal greening to turn to evergreen and colorful one; Building the mountains by piling of discarded soil enables the plane and straight greening turn to the three-dimensional and curved one. Meanwhile, the three-dimensional development of the high-density central area makes full use of the facilities of the green space and its underground space to achieve the combination of concentration, evacuation and disaster prevention.

For example, in the center of La Défense in Paris, in order to provide a good walking and landscape environment based on the high-intensity development, an oversized roof garden was built in the form of an overhead platform. The roof garden is a pure park and walking system, while the ground floor is connected to the urban transportation network, such as roads and public transportation, which completely achieves the diversion of people and vehicles. (Fig.3)

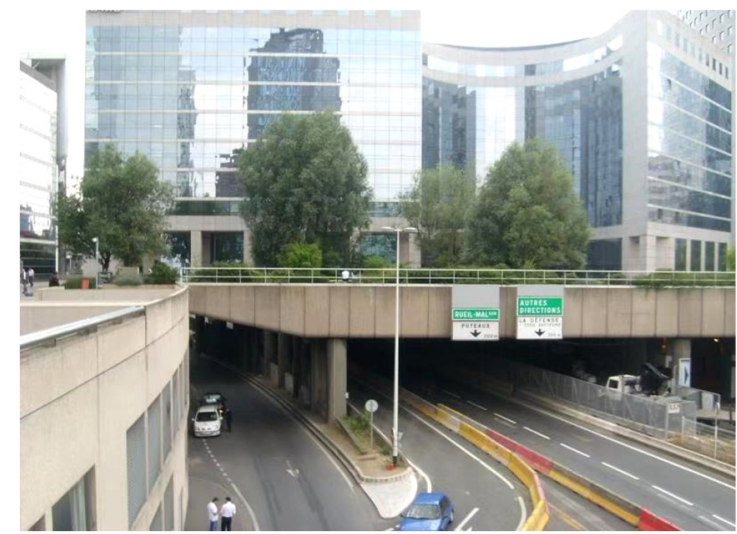

Fig. 3. Three-dimensional development of the open space in the centers of La Défense, Paris

\section{Open space for commerce}

The open space for commerce in urban centers is the outdoorization of commercial services, cultural and recreational facilities, which interweaves leisure pedestrian traffic and commercial services. The open space for commerce includes commercial pedestrian streets (districts), commercial squares and commercial unilateral streets. The commercial pedestrian street is an open space complex guided by pedestrian traffic, restraining the transportation of motor vehicles and bicycles. It consists of retail business group as main facilities, catering service group as auxiliary facilities and 
cultural and entertainment as related facilities. The commercial square has main functions of commercial entertainment, located on the partial setback of large buildings or the street where the roads meet. The commercial unilateral street is a linear walking space for commercial and leisure activities, formed by the road unilaterally setback, retaining the traffic function of the motor vehicle.

\subsection{Typical mode of open space for commerce}

Commercial pedestrian street is the most typical mode of open space for commerce. Taking people as the core, it enhances the human environment and the sense of shopping security in urban centers, supports the business activities, maintains the organic vitality, and promotes the development of commercial services and cultural entertainment. Commercial pedestrian street is composed of two sides of the building facade and the ground, including commercial building facades, iconic landscapes, floor coverings, merchandise windows, advertising posters, street essays, lighting facilities, plant configurations and event venues.

Commercial pedestrian street has many advantages. From the perspective of business layout, pedestrian zone brings greater flexibility in business layout, restores the dominant position of people in transportation, and provides people with high-quality activity space and communication place. From the view of space, without the interference of the car, commercial pedestrian street creates a different landscape, attracting citizens and tourists to stay and easily walk or stop in the pedestrian street, then the beauty of the building, the street, and the city historical context can be discovered. From the point of activity management, the commercial pedestrian street provides a safe shopping place, which allows consumers and operators not to worry about the pressure of traffic safety and noise interference. Therefore, it is conducive to attracting a large number of people, driving the development of business and bringing more economic benefits to commercial facilities around the pedestrian street.

\subsection{Historical evolution of open space for commerce}

The open space for commerce in urban centers has undergone more than half a century of evolution, from the early commercial space of the early 1940 s to the comprehensive activities site with modern function integrated business, culture, recreation, socializing, etc. Although its main function is still commerce, the relationship with the pedestrian has completed the transition from estrangement to solicitude, and finally adapted to the wide range of public activities required by them. (Fig.4).

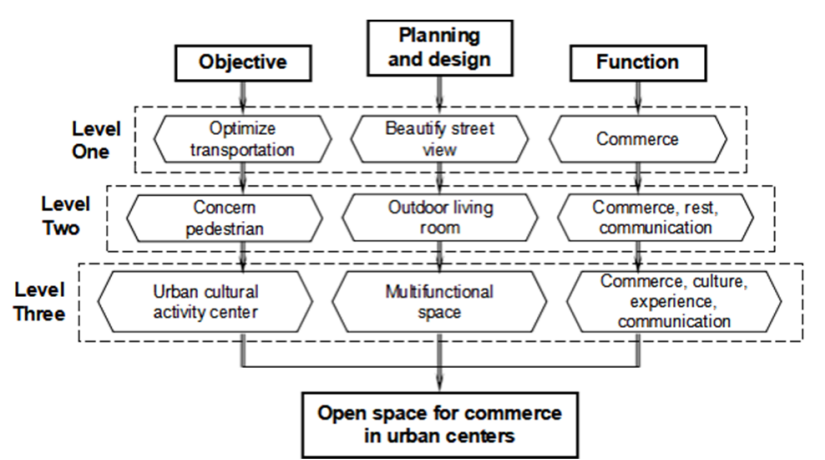

Fig. 4. Historical evolution of open space for commerce

\subsection{Cultivation method of open space for commerce}

First, a successful planning of open space for commerce must focus on synchronizing with the level of urban economic development and the scale of commercial consumption. When the pedestrian street completely abandons motor vehicle traffic, it also means abandoning the pass-through consumption it brings, and fully supporting the huge all-hour commercial consumption with reaching consumption, which has high requirements for the economic development level of the city. In spite of their own level of economic development, some small cities have forcibly turned the main road into a commercial pedestrian street at a huge cost. As a result, the consumption of people is insufficient, which restricts the development of urban centers. Pedestrianisation of commerce can be done in a variety of ways. Complete pedestrianisation is not the only means. Partial pedestrianisation and commercial unilateral streets are good ways to cultivate a open space for commerce.

For example, during the commercial development period of the 1990s, Hunan Road was set as a pedestrian market at the night time of weekends and holidays through traffic control, which reasonably solved the contradiction between commuting and shopping. At the same time, the complete pedestrianisation in the Lion Bridge section, as a food and beverage street, forms functional and environmental differences with the commercial retail along the road, enriching the consumption levels of Hunan Road. (Fig.5)

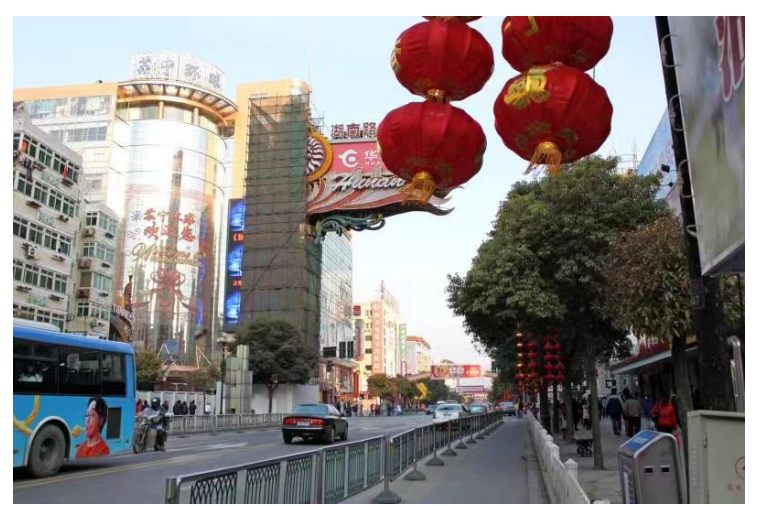

Fig. 5. Time-limited pedestrianisation of Hunan Road in Nanjing

Second, as for the layout of the facility, it is necessary to consider the continuity of the commerce and reduce the 
"blank area" of the commercial fault. If the residential or office building must be set up in it, the bottom layer should be set as commerce to ensure the continuity of the shopper flow. The open space for commerce is mainly for the commerce with high visitor flow-rate, which requires the commercial atmosphere to reflect the excitement and prosperity, so the administrative, educational and other buildings, whose serious atmosphere will affect the overall style of the commerce, should be less inserted in it.

Third, with the consideration of public activities and gathering activities, the open space for commerce should be based on hard paving, supplemented by greening, setting wide paving and exquisite landscape to buffer and guide. The greening mainly should be large potted trees and shrubs, which is flexible for layout, while the covering soil greening should be large trees that can provide the shade, combining with the landscape for rest to provide public rest site.

Fourth, use the dimension of which regards human as the main body of design. The pedestrian street should not be too long, as far as possible within 500 meters. If it exceeds this length, the similar business forms should be concentrated and formed into functional relative partitions, which is convenient for the public to choose the shopping area suitable for them. Due to the demand for shopping and business operations, the open space for commerce should not be too large, so that the display window and advertising sign of opposite shop scan be seen. However, the squares should be appropriately enlarged.

The last, carry forward the regional cultural characteristics. On the layout, even if the format of modern business is dominant, in a certain extent, the spatial form of local features should be inherited. For example, the commercial pedestrian street in Jiangyin, at the location of the county school, still retains the remaining building. Therefore, the planning of the main square on the pedestrian street reflects the main landscape of this history, with the theme of the "Examination results announced", designing the group sculpture, which can not only play the leisure and recreation role, but also can carry the historical context and enhance the cultural connotation. (Fig.6) The main building of Guangzhou Shangxiajiu Pedestrian Street adopts the typical historical mode of Lingnan commercial architecture, named the Arcade. It can be well shaded and sheltered from rain, suitable for the southern climate, and also enrich the spatial hierarchy of pedestrian street. (Fig.7)

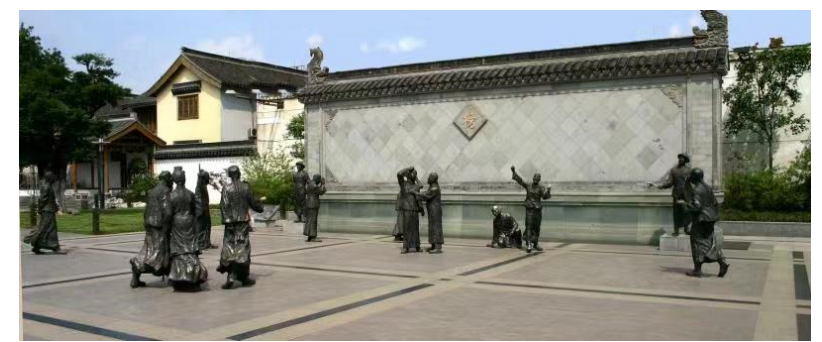

Fig. 6. The theme square of Jiangyin commercial pedestrian

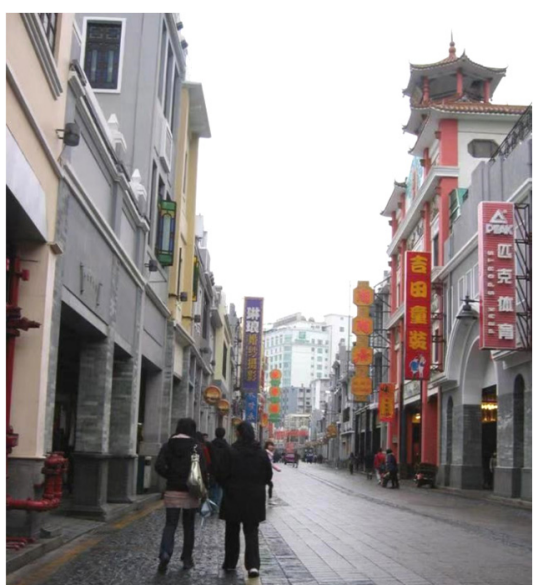

Fig. 7. The Arcade of Guangzhou Shangxiajiu Pedestrian Street

\section{Open space for transportation}

The open space for transportation in urban centers is based on the traffic organization, supplemented by ecological and landscape appreciation function, including station square, traffic green island square and traffic isolation green space.

Station Square, located around the train station, provides functions such as stop, transfer, and diversion for large-scale crowd distribution. It mainly solves the problem of traffic distribution and the connection with urban centers, using the three-dimensional traffic mode to quick diversion, such as underground passages and flyovers.

Traffic Green Island Square is a green traffic island set up at the intersection of several main roads to provide traffic guidance and landscape appreciation. For example, the Xinjiekou Traffic Island in Nanjing is an important traffic diversion node at the intersection of four main roads in downtown of Nanjing. To commemorate Mr. Sun Yat-sen, the bronze statue is set in the center of the square. It is expanded into a circular island with a diameter of 8 meters, paving a one-meter road around the outer island and surrounding green landscape. (Fig.8)

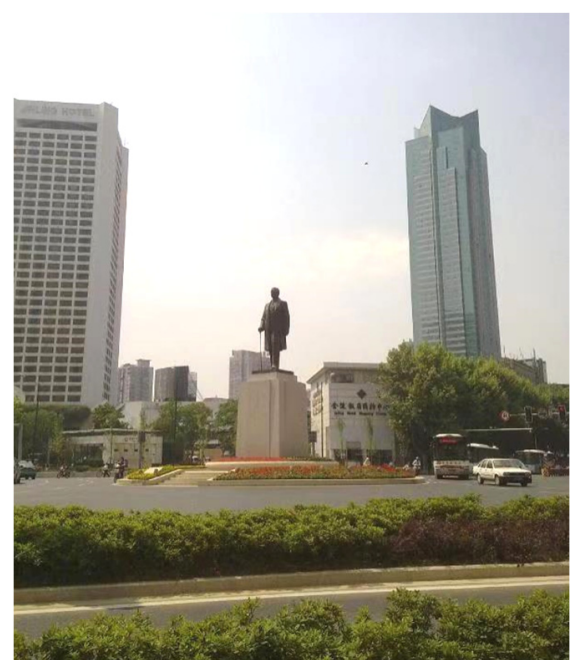

Fig. 8. Xinjiekou Traffic Island in Nanjing

Traffic Isolation Green Space is a protective green block, located on both sides of express artery or elevated roads, providing functions like noise mitigation, safety 
protection, and landscape ecology. Due to the dense construction and pedestrian flow in urban centers, planting in isolated green spaces should aim at landscape isolation and noise isolation. For example, Beijing Chaoyang East Third Ring Road, as a city expressway, has no good green separation zone, so that the functions of landscape and noise reduction are not reflected. The city construction land is close to the East Third Ring Road, and there is almost no protective green space between them, which results in poor ecological effect and greater traffic noise impact on the surrounding buildings. (Fig.9)

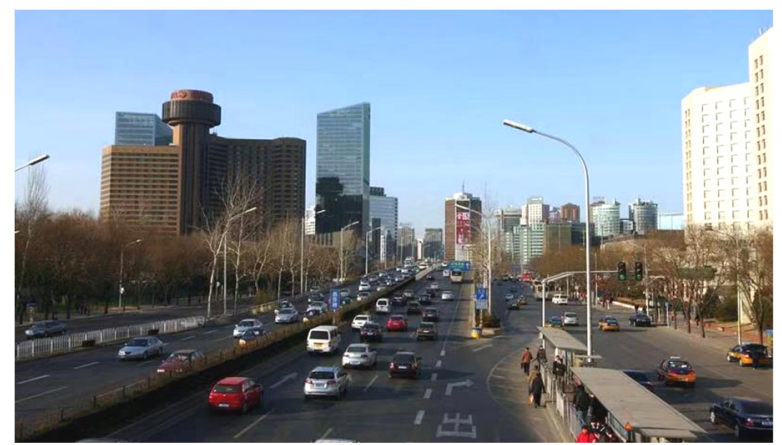

Fig. 9. Beijing Chaoyang East Third Ring Road

\section{Planning points of the open space system in urban centers}

The open space in urban centers is not only the element to beautify the landscape of the urban public center, but also exercises multiple functions and reflects cultural values. The rational planning and design of the open space in urban centers is the protection of the ecological environment, the advancement of the public activities and the improvement of the life quality, and also the promotion of urban diverse business culture. As an important part of the orderly development of urban centers, how to make a rational planning to protect the open space is related to the ecological environment protection of the high-density core areas of the city; it is related to the advancement of the public activities and the improvement of the quality of public services; it is related to the continuation of urban business culture and historical context.

\subsection{Open to the public}

The service target is intensive flow of citizen who arrives in urban centers, so it has a considerable scale, on the contrary, small-scale green space is meaningless. Index control is a common method in the planning of open space, especially the Green Space. Inserting open space in the shadow area of urban centers is a qualitative method, then the index that larger than 1 hectare is a quantitative method to achieve open space to play its due role. The open space should not be enclosed, and should be opened to the public.

\subsection{Easy to reach}

As the conversion space in or out of urban centers, its main function is to provide the distribution and relaxation space for the tourists, so it must have good accessibility. The conversion includes two aspects. One is the conversion of urban public transportation and the central pedestrian system, that is, the connection between the central area and the city. The other is the conversion of the interior and exterior space of the large service facility building, that is, the connection between the open space and the surrounding area. The open space is usually connected to the urban public transportation stations, which is convenient for the distribution of a large number of people. At the same time, it is connected with the main facilities through corridor, pedestrian bridge or underground square, providing the public with a rest space.

\subsection{Associate with landscape}

Although the open space in urban center requires a certain amount of scale, due to the high land price and the cost of demolition, per square meter open space is very precious. The planning design that connects the large natural elements in the visual landscape, opens the visual corridor and produce landscape effects, like "Scenery-borrowing", "Scenery-viewing" and "Vista-liner", is a very effective. The urban large natural elements, including rivers, lakes and hills, are the most beautiful areas of the city and maintain the richest historical and cultural remains. Therefore, the open space urban centers can be linked with the natural landscape system, becoming an extension of the urban large-scale greening system, and becoming a concentrated display window of urban characteristics, which obtains the maximum landscape benefits with the smallest scale.

\subsection{Compound utilization}

Due to the scarcity of land and high-density flow in urban centers, the open space is compounded, which is manifested in several aspects: compound function of recreation, retail service, cultural entertainment, green ecology, etc.; compound element including architectural design, street layout, square environment and vegetation arrangement, etc.; compound development of multidimensional space including open space underground parking and three-dimensional transportation, etc.; compound ecological effects of water surface, woods, grassland and hard squares.

\subsection{Economic construction}

Planning methods, such as Incentive Control and Development Right Transfer, mostly be used in the construction of open space in urban centers. These applications in the United States, Japan and other countries have become very common.

Incentive Control is a means of reward for encouraging developers to improve the building quality and contribute to the urban environment. Under the premise that the development effect is effectively 
controlled, if the developers can build more public space or public facilities than the required, such as public squares, viewing platforms, parking lots, walking trails, etc., the corresponding level of policy rewards, like increasing plot ratio, reducing fee, relaxing height limit, etc., can be obtained as compensation.

Development Right Transfer, also known as "Plot Ratio Transfer" or "Air Power Transfer", arises in the United States and is a spatial guide for regulating public and private interests. Due to the need for public activities, the development of these plots is restricted, so that the undeveloped space rights over these land are allowed to be transferred to the adjacent ones, and the developers who have lost building area in the green parks and activity squares can be compensated at other bases nearby. This kind of compensation guarantees the interests of the developers, and also takes into account the construction requirements of the open space in urban centers, and plays a significant role in urban planning management.

The center of Singapore Bay-Orchard has formed a typical high-intensity development pattern due to the complex terrain and the harsh conditions of land use. Under this circumstance, a large number of open spaces such as green spaces parks are still being built and open to the public. These open spaces are mostly connected directly to the subway and bus stations, which brings high accessibility, and are connected with the core area three-dimensional walking system, providing functions such as rest and appreciation, greening and ecology, etc., and are partially combined with cultural facilities such as museums and exhibition halls, providing culture and entertainment features. In this way, the green open space becomes an important activity hub for the entire urban centers. (Fig.10)

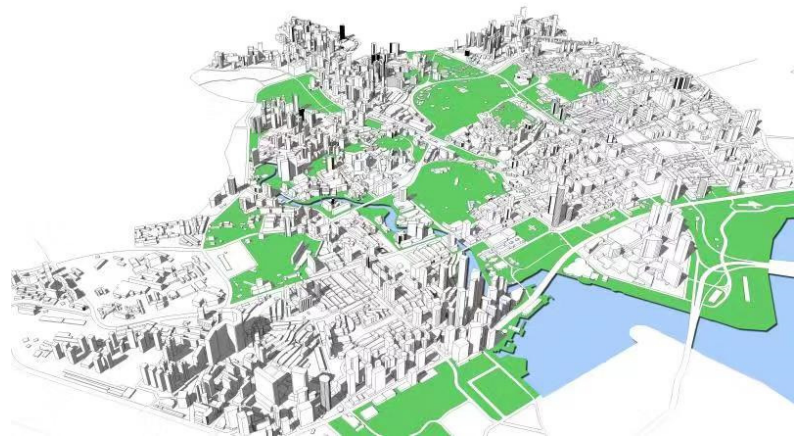

Fig. 10. The open space system in Singapore Bay-Orchard centers

However, as the largest city square in Asia, Dalian Xinghai Square, an oval square with an area of 1.1 million square meters, located in the southern coastal scenic area of the city, does not bring good economic benefits, although built a good open space with coastal landscape.

For the open space of such a scale, the construction intensity of the surrounding land should be improved to realize the utilization efficiency of the land, but the construction around Xinghai Square is still relatively low density, resulting in waste of land resources and lack of space aesthetics corresponding to large-scale squares. In terms of its function, it is merely for viewing, without retail commercial, cultural and recreational facilities and rest facilities, and does not play an ecological role in communicating the natural resources and the city. The square is made of lawns and hard grounds, without tall arbor greening, making the overall space too empty, so that visitors are completely exposed to the sun. Therefore, the square is used in low efficiency, lost its role in gathering popularity. (Fig.11)

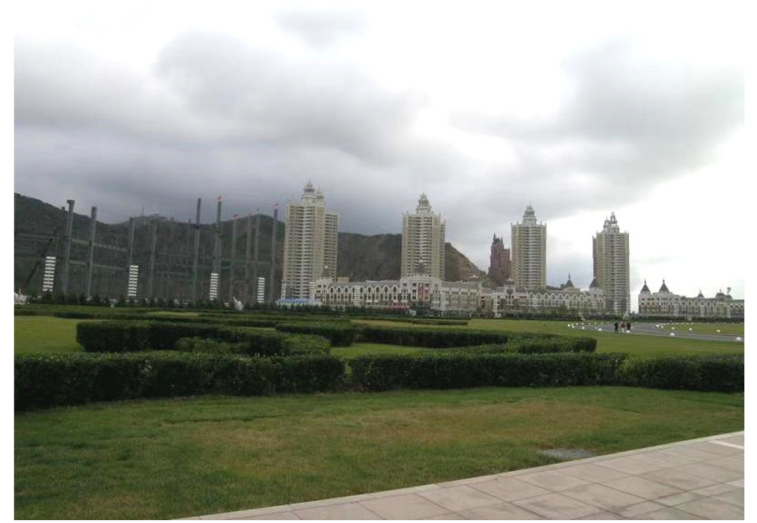

Fig. 11. The open space in Dalian Xinghai Square centers

\section{References}

1. N.J. Greenwood, Z.G.Liu, et al., Human environment and natural systems (Chemical Industry Press, Beijing, 1987)

2. S.Takahara, Z.Z.Yang, et al., Urban green space system (China Architecture \& Building Press, Beijing, 1983)

3. C.Alexander, X.H.Zhou, et al., Architectural Pattern Language (Intellectual Property Publishing House, Beijing, 2002)

4. A.Heckscher, P. Robinson, Open Space The Life of American Cities (Hager, New York, 1977)

5. Q.Yu, The Construction of Modern Urban Open Space System. Urban Planning Forum 1988(6): 49-56

6. J.W.Lu, Z.Zheng, Urban Design and Development. Architectural Journal 1997(4):6-8

7. C.H.Zhang, People, Open Space and City (China Architecture \& Building Press, Beijing, 1990)

8. J.G.Wang, Urban Design. Version II (Southeast University Press, Nanjing, 2004) 